\title{
Digital breast tomosynthesis: A state-of-the-art review
}

\author{
Marcello Alessandro ORSI ${ }^{1}$, Cellina $\mathrm{M}^{1 *}$, Rosti $\mathrm{C}^{\mathbf{1}}$, Gibelli $\mathrm{D}^{2}$, Belloni $\mathrm{E}^{3}$ and Oliva $\mathrm{G}^{\mathbf{1}}$ \\ ${ }^{1}$ Department of Radiology, Ospedale Fatebenefratelli, ASST Fatebenefratelli Sacco P.zza Principessa Clotilde 3, 20121, Milano, Italy \\ ${ }^{2}$ Dipartimento di Scienze Biomediche per la Salute, Università degli Studi di Milano, V. Mangiagalli 31, Milan, Italy \\ ${ }^{3}$ Department of Radiology, Civil Hospital, Corso Milano 19, 27029, Vigevano, Italy
}

\begin{abstract}
Digital Breast Tomosynthesis (DBT) is an X-ray mammography technique where multiple low-dose projection images of the breast are reconstructed in multiple tomographic images creating a semi-3D mammogram. This enables the visualization of a sequential set of thin sections of the breast, overcoming the masking effect of overlying fibroglandular breast tissue, then improving carcinoma detection and reducing false-positive cases. This review aims at describing current DBT technique, analyzing DBT in clinical practice and providing an overview of published studies on clinical experience with DBT in the screening and diagnostic settings.
\end{abstract}

\section{Introduction}

Screening asymptomatic women for breast cancer with mammography can reduce cancer-related mortality up to $30 \%$, since breast carcinoma is a progressive disease and early detection enables better prognosis [1-7]. However, evidence suggests that $15-30 \%$ of breast cancers are missed by standard screening mammography, and this rate can be even higher in women under 50 years [8] and in women with dense breast [9-12]. In fact, the primary limitation of mammography is the overlapping of dense fibroglandular breast tissue that can decrease the visibility or hide underlying malignant lesion [13-16].

Moreover, the overlay of normal fibroglandular breast tissue may mimic the appearance of carcinoma, leading to a reduction in specificity and increase in false-positive recalls [16]. In the early 2000s, mammography has transitioned from film-screen system to digital detectors $[17,18]$, with an improved diagnostic performance, especially in women with dense breasts [19]. The introduction of full field digital mammography (FFDM) has also allowed the development of new diagnostic breast techniques like digital breast tomosynthesis (DBT).

DBT is an X-ray mammography technique where multiple low-dose projection images of the breast are acquired moving the X-ray tube in an arc over a limited angular range, and subsequently reconstructed in multiple tomographic images creating a pseudo-3D mammogram $[20,21]$. Such acquisition enables the visualization of a sequential set of thin sections of the breast, allowing overcomes the masking effect of overlapping fibroglandular tissue, improving carcinoma detection and reducing the number of false-positives [21].

This technique showed better sensitivity and specificity compared to FFDM, especially for detection of non-calcified breast cancer [22] and it has been proven to be an efficient tool either in screening and diagnostic settings, since its introduction in the clinical setting after the approval by the Food and Drug Administration (FDA) in 2011.

DBT provides pseudo-3D images allowing a more detailed delineation of breast lesion contour, enhancing the visualization of distortions and also providing an accurate localization and extension of breast lesions, in addition to possible identification of additional cancer foci [23]. This article describes the current available DBT technique and provides an overview of the state of the art in both screening and diagnostic clinical settings.

\section{Technique}

The breast is compressed and held between the compression paddle and the detector, as in performing FFDM. The $\mathrm{x}$-ray tube moves across a limited arc above the breast, acquiring a sequence of low-dose exposures at preset intervals, each from a different angle, resulting in a series of projection images [24-26]. The angle range (from 15 to $50^{\circ}$ [20]), the tube motion, the arc length and the time needed to obtain a whole set of projection images vary among different manufacturers [27,28] (Table 1).

In the post processing, raw data from DBT projections can be reconstructed to obtain a series of images of the whole breast, typically with $1 \mathrm{~mm}$ spacing, parallel to the DBT platform plane [29]: this process allows a pseudo-3D evaluation of breast tissue distribution. Due to the limited angle of the projections, DBT has a high spatial resolution in the plane parallel to the detector, but a lower spatial resolution in the perpendicular direction [28]; however, spatial resolution in the depth direction is sufficient to reduce the effects of breast tissue overlapping [29,30].

The tomographic slices are then displayed on the tomosynthesis vendor's proprietary workstation or PACS for radiological interpretation; the total number of reconstructed images depends on the thickness of the compressed breast and is equal to the thickness of patient's breast plus five additional slices, that are added to each set of

${ }^{\star}$ Correspondence to: Michaela Cellina, Ospedale Fatebenefratelli, Radiology Department, P.zza Principessa Clotilde 3, 20121, Milano, Italy; Tel: +3963632424; Fax: +3963632260; E-mail: michaela.cellina@asst-fbf-sacco.it

Key words: digital Breast Tomosynthesis; mammography; screening; breast cancer; birads

Received: December 07, 2018; Accepted: December 20, 2018; Published: December 26, 2018 
Table 1. Characteristics of Clinical DBT Systems

\begin{tabular}{|c|c|c|c|c|c|}
\hline Manufacturer & HOLOGIC & GE Healthcare & $\begin{array}{c}\text { Siemens } \\
\text { Healthcare }\end{array}$ & FUJI & $\begin{array}{c}\text { Internazionale Medico } \\
\text { Scientifica }\end{array}$ \\
\hline Model & Selenia Dimensions & Seno Claire & MAMMOMAT Inspiration & Amulet Innovality & Giotto \\
\hline Scanning Angle $^{\circ}$ & 15 & 25 & 50 & $\begin{array}{l}40 \mathrm{HR} \\
15 \mathrm{ST}\end{array}$ & 20 \\
\hline Projection & 15 & 9 & 25 & 15 & 13 \\
\hline Scanning Time (sec) & 4 & 7 & 24 & $\begin{array}{l}9 \mathrm{HR} \\
4 \mathrm{ST}\end{array}$ & 12 \\
\hline Tube Motion & continuous & step and shoot & continuous* & continuous & Healthcare \\
\hline Detector Material & a Se & a Si/Csl & a Se & $\mathrm{aSe}$ & a Se \\
\hline Reconstruction Algorithm & FBP & Iterative & FBP & FBP & Iterative \\
\hline
\end{tabular}

FBP $=$ Filtered Back Projections

$\mathrm{ST}=$ Standard Mode

$\mathrm{HR}=$ High Resolution Mode

a $\mathrm{Se}=$ amorphous selenium

a $\mathrm{Si} / \mathrm{Csl}=$ amorphous silicon/cesium iodine

*Continuous scanning mode with short pulses of radiation

images to the image non-receptor side to help define the breast edges. The reader can scroll through the images manually or in a dynamic cine mode, and change the section thickness.

The image quality of DBT is highly dependent on the geometry of the system and on the choice of optimal acquisition, reconstruction, and display parameters.

\section{Dose}

DBT images can be acquired by using an FDA-approved combined acquisition mode [31] that is FFDM and DBT performed during the same examination: DBT projection images are carried out first, and then standard mammographic images are obtained in a perpendicular position, with no modifications of breast compression, or in an independent acquisition.

DBT images are low-dose, with a mean glandular doses (MGD) of an average-sized breasts of about $2.3 \mathrm{mGy}$ per view, which is about 1 to 1.5-times higher than the dose per view for FFDM [32]; the use in combination of FFDM and DBT doubles approximately the radiation dose [33], therefore, the radiation exposure is one of the main concerns of DBT, especially when using DBT in any program of population screening, also considering that radiation dose is cumulative over time.

In a phantom study on radiation exposure from DBT, Feng and Sechopoulos [34] demonstrated a MGD range of $0.309-5.26 \mathrm{mGy}$ for a single caudocranial view acquisition in FFDM mode and of 0.657 $3.52 \mathrm{mGy}$ for the DBT mode acquisition; for a breast with a compressed thickness of $5 \mathrm{~cm}$ and $50 \%$ glandular fraction, the MGD of DBT was only $8 \%$ higher than that of FFDM acquisition $(1.30$ and $1.20 \mathrm{mGy}$, respectively, per view), while, for a breast with a compressed thickness of $6 \mathrm{~cm}$ and glandular fraction of $14.3 \%$, the DBT resulted in a $83 \%$ higher MGD compared to FFDM (2.12 versus $1.16 \mathrm{mGy}$ ): therefore the range of radiation dose is highly variable, depending on breast size and composition.

MGD is also strongly influenced by some characteristics of the DBT system such as detection process (direct/indirect), scan angle, number of projections, tube motion and reconstruction algorithms, with a large variability among the investigated systems [35], but in any case the dose remains below the FFDM threshold limits established by the European Reference Organization for Quality Assured Breast Screening and Diagnostic Service [36].

The DBT examination may be performed using different combinations [21,37], including 2-view DBT plus 2-view FFDM or single-view DBT plus single-view FFDM $[38,39]$, and varies among vendors; the use of a single-view DBT reduces the MGD [39-42]. Acquisition of standard mammogram is needed to have a panoramic view of the breast and for comparison with previous and future studies, so there is wide consensus that the only DBT images are not enough.

To overcome the dose issue, manufacturers have developed a synthesized mammography (SM) that provides the benefits of a combined FFDM plus DBT examination with a reduced radiation exposure; this is a synthesized 2D image generated from the DBT data. $\mathrm{SM}$ is a maximum intensity projection (MIP) of frequency-weighted reconstructions created by concentrating the tomosynthesis image set to a single 2D image [43]. In May 2013, the FDA approved the use of $\mathrm{SM}$ in combination with DBT to reduce the total radiation exposure by approximately $45 \%$ [43].

SM is now considered a valid alternative for FFDM: its aim is to provide the $2 \mathrm{D}$ component of the DBT examination, particularly useful when comparing with previous [44]. In SM, algorithms preserve imaging characteristics such as microcalcifications and spiculations but with poorer overall resolution and increased image noise [45]. Recent studies showed that the screening performances of SM plus DBT are not inferior to those achieved with FFDM plus DBT [46] and it is likely that SM will replace FFDM in the near future, with a remarkable dose reduction.

\section{Additional reading time}

The interpretation of a DBT examination consists of the evaluation of the $2 \mathrm{D}$ mammography plus the analysis of the DBT dataset, with a variable number of slices, depending on patient's breast thickness: the largest number of images to read in comparison to the only FFDM causes an increase of the interpretation time. Studies have shown that interpretation time for $2 \mathrm{D}$ plus DBT is about twice that of conventional mammography [47]. Skaane et al. [48] reported reading times of 45 seconds for conventional mammography and 91 seconds for DBT; Wallis et al. [40] confirmed an approximate doubling in the reading time with an average time of 67 seconds for conventional mammography and of 124 seconds for DBT. Zuley et al. [49] described an increase in reading time of about $33 \%$.

The extra reading time will obviously impact on radiologist's activity and has a significant relevance in high workloads of screening examinations, but the increased time for images interpretation could be potentially compensated by time saved in the workflow management, 
as already reported $[48,50-55]$, for instance reducing the recall rate in screening setting.

\section{Information Technology storage and connectivity}

Information technology (IT), image storage, retrieval systems and workstation requirements are important issues when introducing DBT in clinical practice $[21,55]$. DBT images require a large amount of storage space, especially in screening programs, due to the high numbers of participating women and considering that storage requirements will increase with time as more patients will have previous DBT studies for comparison.

DBT image files size are 10-20 times that of 2D conventional mammography; the number of slices for each DBT set depends on the breast thickness, but an average combined DBT/FFDM study is about $1 \mathrm{~GB}$ of data, which could be reduced to approximately $250 \mathrm{MB}$ if the DBT images are stored with a 4:1 reversible (lossless) compression $[40,55]$.

Before introducing DBT in clinical practice, it is also important to account that workstation requirements for reading tomosynthesis could be different from 2D FFDM, since standard workstations may not have sufficient random access memory to display DBT. Workstations for DBT analysis require all the standards needed for FFDM reading (i.e.: high resolution monitor, customized visualization protocols, image resizing tools, image annotation, computer-aided detection marks), but also rapid scrolling, cine capacity and the possibility to visualize $2 \mathrm{D}$ and synthesized 2D and DBT images [21].

\section{Clinical applications for DBT}

In clinical practice, 2 sets of DBT are usually acquired for each breast: the cranio-caudal projection and the medio-lateral oblique projection, as usually performed in conventional mammography. The use of two-view DBT combined with two-view FFDM allows obtaining the best diagnostic accuracy; the availability of 2-view FFDM is useful for the accurate detection and characterization of microcalcifications and comparison with previous [28, 56,57].

In symptomatic patients, presenting with palpable lump, nipple discharge, skin thickening and/or nipple retraction, DBT demonstrated a diagnostic accuracy equivalent or superior to conventional mammography $[58,59]$ and some studies stated that DBT had the advantage to replace additional spot compression mammography, having the same diagnostic accuracy, and to reduce the use of other diagnostic techniques, such as ultrasound and MRI [58-61]. Moreover, recent studies demonstrated that DBT in the diagnostic settings decreased the number of lesions classified as BI-RADS category 3 , particularly masses and asymmetric lesions, with a substantial increase in the number of those categorized as BI-RADS 1, 2, or 5 [62].

In the screening setting, DBT plus FFDM versus FFDM alone showed overall improved accuracy [47], especially in women with scattered and heterogeneous breast densities [63]. Particularly, DBT can provide benefits in some diagnostic contexts: as lobular carcinoma, that is often difficult to detect on FFDM due to its linear pattern of growing without a typical mass shape (Figure 1). Also breast cysts and fibroadenomas are better identified and characterized by DBT than FFDM because their shape and margins are generally better defined in the DBT images as oval or round circumscribed masses with smooth margins. Dermal and vascular calcifications, surgical scars and intramammary lymph nodes are other common findings, better visible and adequately assessed by DBT [64] (Figure 2).

Due to its greater sensitivity for benign lesions, the introduction of DBT in a FFDM-based screening could lead to a recall rate increase related to previously undetected benign masses; for this reason, the overall screening recall rate reduction, seen with the implementation of DBT, is primarily due to more accurate assessment of summation shadows and pseudo lesions [21].

Malignant tumors can be better detected by tomosynthesis when compared with FFDM alone; the standard DBT views show precise details of the tumor, highlighting suspicious irregular shapes, indistinct or spiculated margins and other important features, particularly in cancers surrounded by normal fibroglandular tissue (Figures 3-6).

\section{Staging}

Mammographic evaluation of breast cancer should include lesion size, number of lesions, and the assessment of contralateral disease. DBT is a valuable tool for breast cancer staging due to its ability to detect multifocal or contralateral disease and its accuracy of lesion measurement. Lesion size evaluation at FFDM can be challenging and usually this feature is better assessed at US or MRI [65]. The tumor is depicted in multiple images at DBT and, only the core nucleus of the mass in the slice with the best cancer visualization should be measured in order to assess the actual tumor size, avoiding the spicules that may be seen on other sections [28]. Several studies have shown that this approach correlates well with pathologic analysis in masses measuring up to $20 \mathrm{~mm}$ and is more accurate than FFDM alone [66]. Due to the possibility of having a greater visualization of details, DBT can depict
A

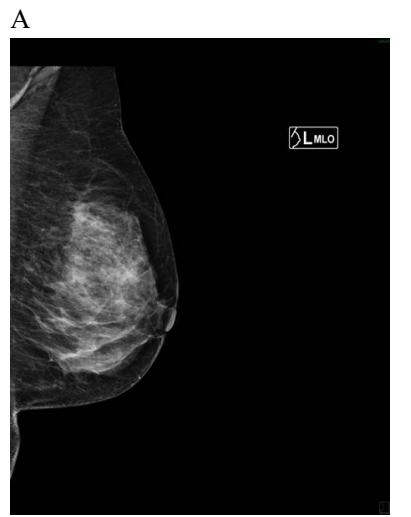

B

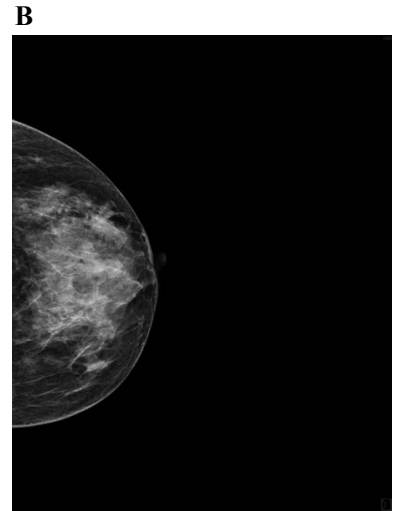

$\mathrm{C}$

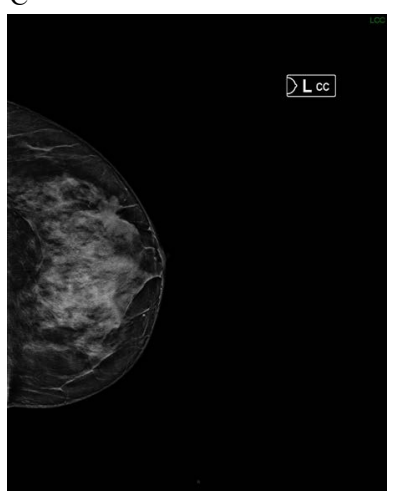

D

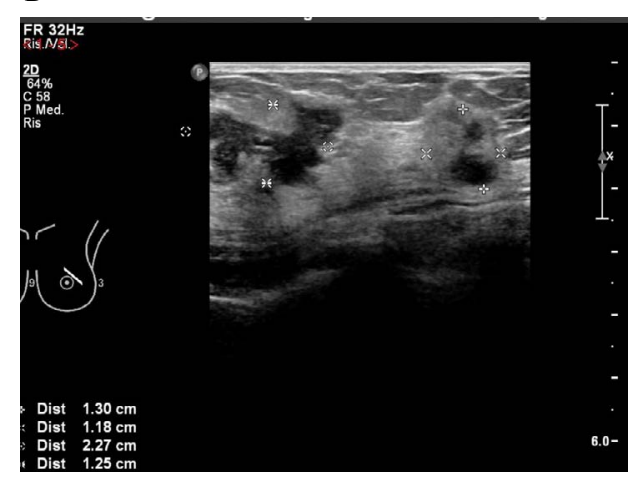

Figure 1. Asymptomatic 67-year-old woman with no family history of breast cancer, with a partially hidden, subtle, irregular shaped mass in the upper external quadrant of the left breast DBT allowed the detection of a $3 \mathrm{~cm}$ high-grade invasive lobular carcinoma. The lesion was measured more precisely on DBT than on ultrasound 1A left MLO FFDM, 1B left CC FFDM, 1C left CC DBT, 1D left US 


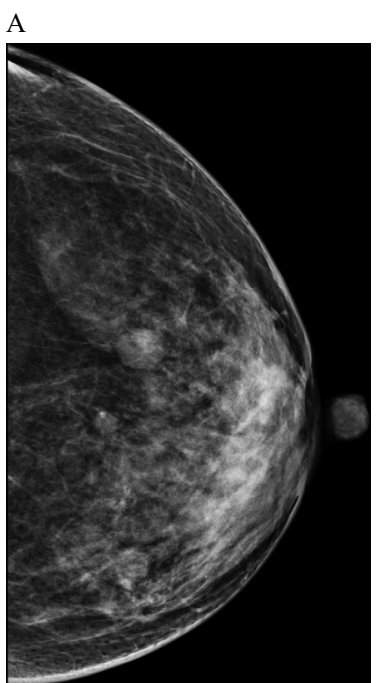

B

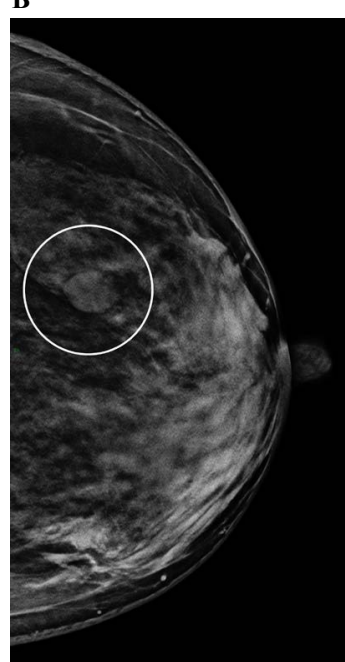

$\mathrm{C}$

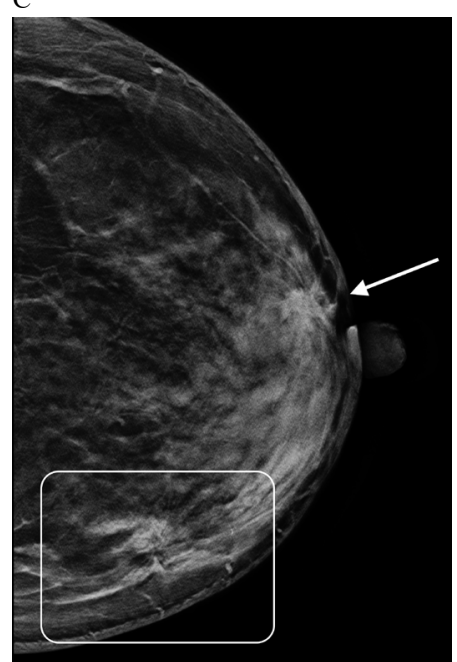

Figure 2. 53-year-old woman with left nipple bloody discharge, I grade family history of ovarian cancer and a personal history of left breast lumpectomy for fibroadenoma. No previous examinations were available

2A left CC FFDC, 2B left CC DBT (at upper quadrants), 2C left CC DBT (at equatorial line)

The circle in 2B indicates a cyst, with smooth, well-defined margins, better depicted in DBT.

The rectangle in $2 \mathrm{C}$ shows the scar of the previous lumpectomy.

The arrow in $2 \mathrm{C}$ indicates a $1.5 \mathrm{~cm}$ mid-grade invasive ductal adenocarcinoma

A

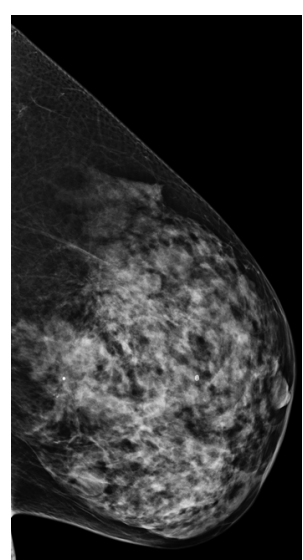

B

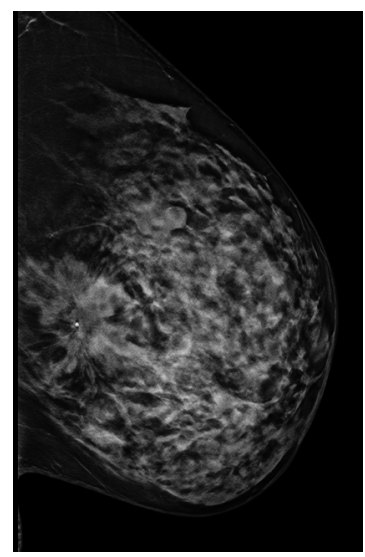

Figure 3. Clear visualization of an architectural distortion at DBT in a 45-year old asymptomatic woman, with no family history of breast cancer, at her first routine screening mammography 3A left ML FFDM, 3B left ML DBT

The presence of a parenchymal distortion, located in the central pre-pectoral breast area, is well visible on DBT, in a heterogeneously dense breast. The lesion was occult on ultrasound and tomosynthesis-guided Vacuum Assisted Breast Biopsy was performed and demonstrated invasive ductal carcinoma with ductal carcinoma in situ

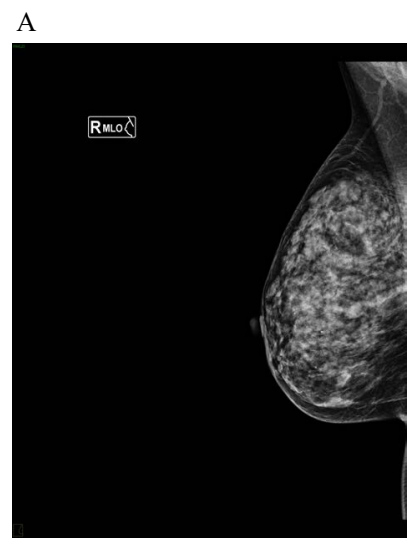

B

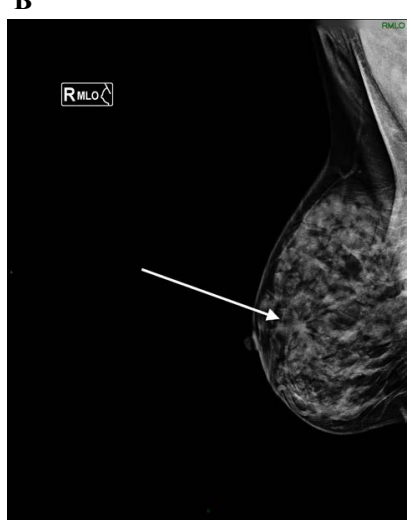

$\mathrm{C}$

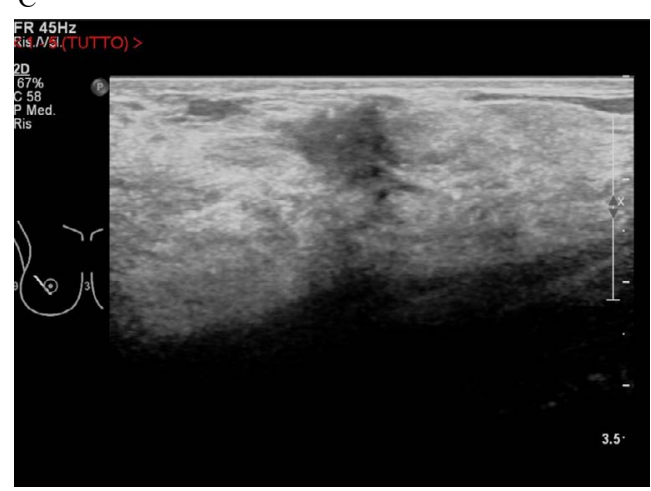

Figure 4. Detection of a small mass at DBT in heterogeneously dense breast, in a 49-year-old asymptomatic woman due to the better visibility of surrounding spicules in DBT images $4 \mathrm{~A}$ right MLO FFDM, 4B right MLO DBT, $4 \mathrm{C}$ right US of the lesion US-guided core needle biopsy proved an invasive ductal carcinoma 

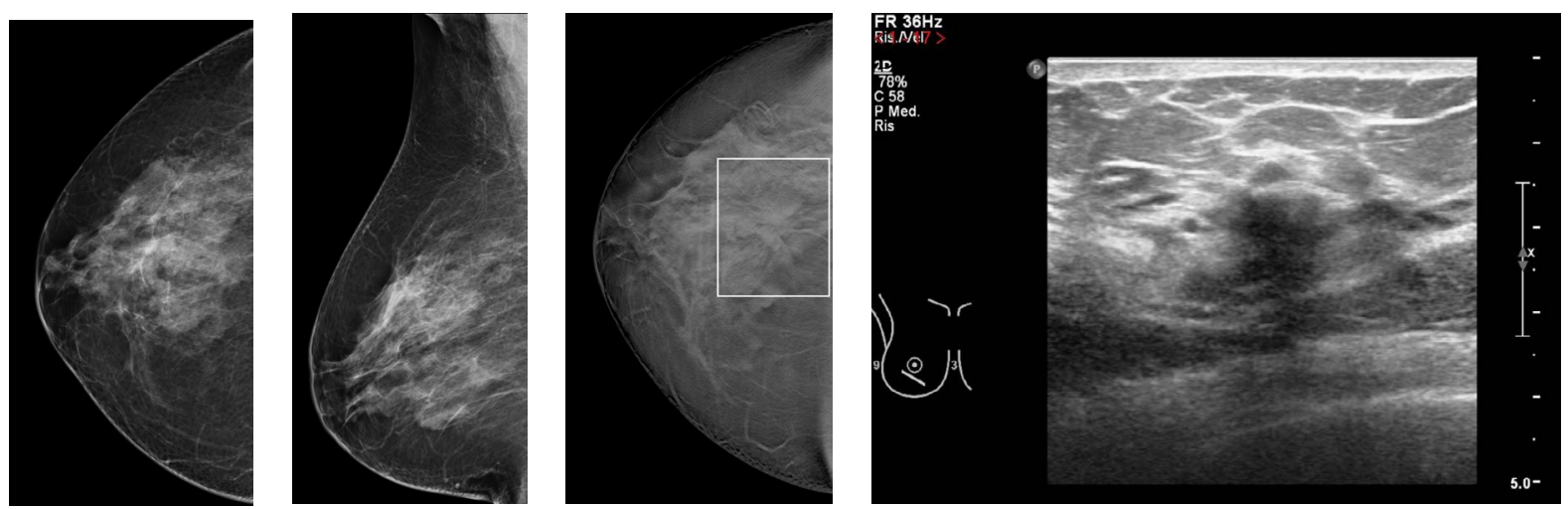

Figure 5. DBT showed a mass associated with an architectural distortion in the periareolar infero-external quadrant of heterogeneously dense right breast of a 45 -year-old asymptomatic woman, with a recent ultrasound diagnosis of ill-defined hypoechoic area, non-palpable at clinical examination

5A right CC FFDM, 5B right MLO FFDM, 5C right CC DBT, 5D right US of the hypoechoic area.

Invasive ductal carcinoma with ductal carcinoma in situ was diagnosed with US-guided core needle biopsy
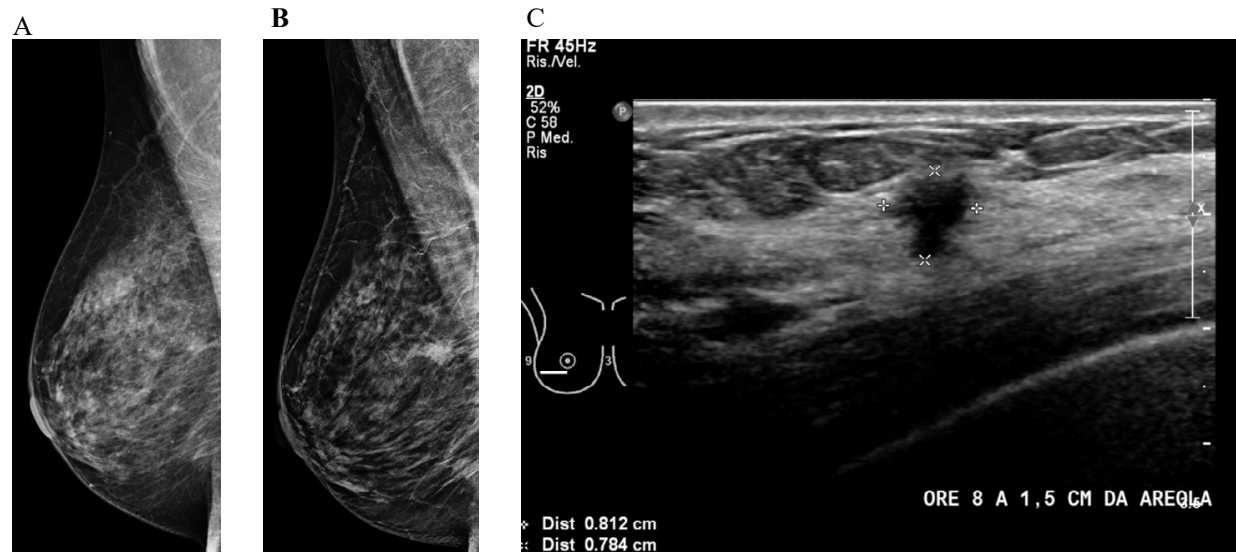

Figure 6. DBT showing a low-density oval mass in a 67 -year-old asymptomatic woman with fatty breast

6A right MLO FFDM, 6B right MLO DBT, 6C right US of the lesion

US-guided core needle biopsy demonstrated low-grade invasive ductal carcinoma

additional satellite lesions, in multifocal disease, that can change patient management (Figure 7). There is evidence supporting that the use of DBT in the evaluation of the contralateral breast in women with newly diagnosed breast cancer could represent a faster, less invasive and more economical way compared to MRI, which now represents the gold standard in detecting contralateral cancer (Figure 8) [28].

In the next section, we provide an overview of the DBT appearance of the most common features related to malignancy.

\section{Architectural distortion}

Architectural distortion is a subtle imaging finding, usually better detected at DBT than at FFDM, frequently recognizable on only one view; it may be similar in density to surrounding tissue and may have areas of associated fat. The detection of architectural distortions is important due to its known positive predictive value for breast cancer $[50,67,68]$. In clinical practice, DBT is useful for the evaluation of potential architectural distortion, allowing a better detection of the radiating lines that converge to a point, especially along a fat-glandular interface and at the apex of the breast, where summation is common [28] (Figure 3). Careful scrolling through a suspected architectural distortion on DBT may also reveal an associated mass previously undetected by FFDM (Figure 5). In a study by Dang PA et al. [57], common presentations of cancers on tomosynthesis were irregular spiculated masses $(61 \%, 105 / 172)$, architectural distortion $(12 \%$,
20/172), and lobulated circumscribed masses (8\%, 13/172). Of the cancers presenting as architectural distortion on tomosynthesis, $50 \%$ $(10 / 20)$ were occult on conventional mammography and $20 \%(4 / 20)$ were characterized as asymmetry or focal asymmetry on conventional mammography. Cancers presenting as architectural distortion on tomosynthesis had a disproportionately higher percentage of ILCs (20\%).

Radial scars, post-biopsy and post-surgical scars can be detectable as architectural distortions and may be better visible at DBT than on FFDM (Figure 2). When an architectural distortion is detected, an accurate review of breast clinical history is needed: without a correlative surgical history, image-guided biopsy of architectural distortion is often warranted because benign and malignant architectural distortions are indistinguishable [50].

\section{Focal asymmetry}

Focal asymmetry is an area of tissue visible on two views in the same breast, unilateral, without a correlate of tissue in the same position of the contralateral breast; it has similar shape on different mammographic projections, but without the conspicuity or the borders of a mass [69]. DBT is useful to confirm and characterize this finding as a true asymmetry, dismiss it as a superimposition, or reclassify it as a mass. In a study by El Maadawy et al. [70] the authors used DBT 

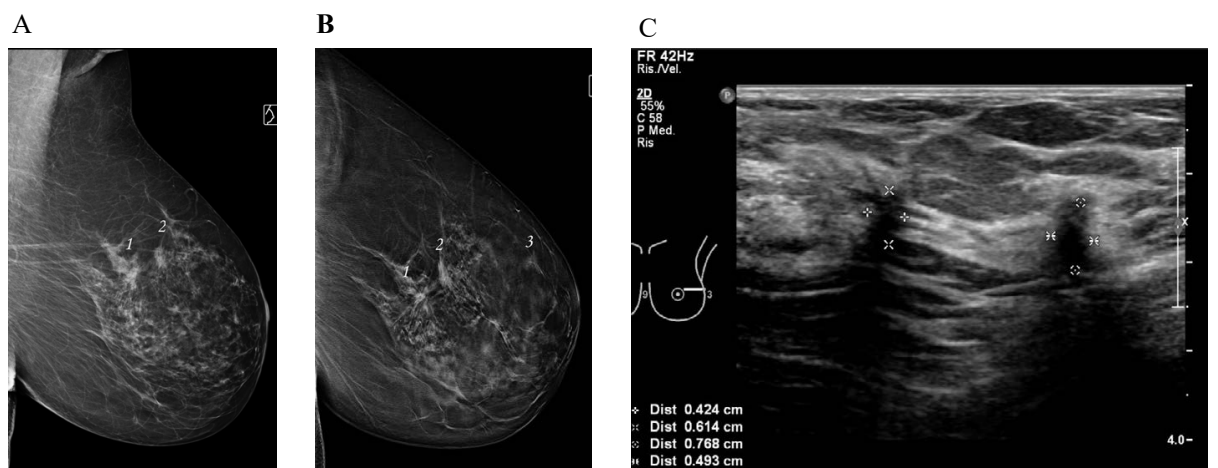

Figure 7. Asymptomatic 79-year-old woman with fatty breast, with two suspicious masses / architectural distortions in the left breast at FFDM 7A left MLO FFDM, 7B left MLO DBT, 7C left US of the two biggest lesions

US confirmed the two lesions visualized at FFDM.

DBT was able to detect another small suspicious lesion in the same breast.

A

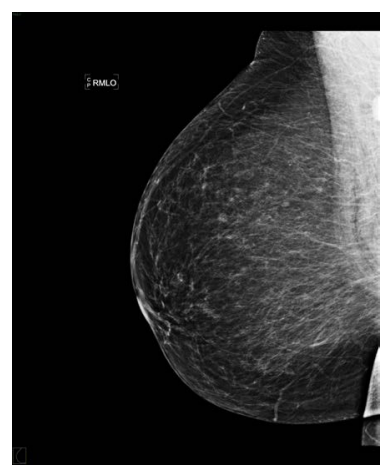

E

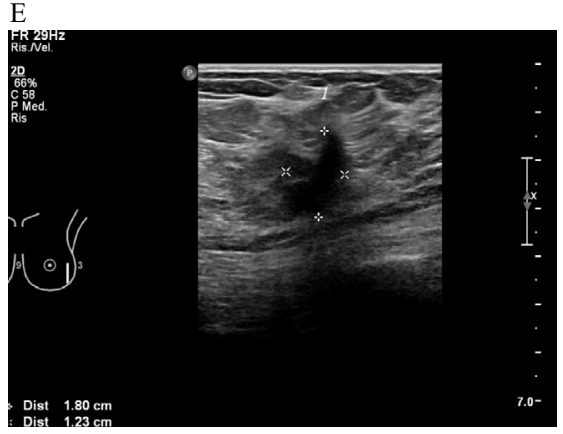

B

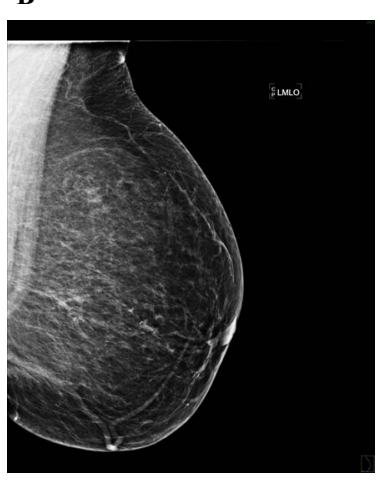

F

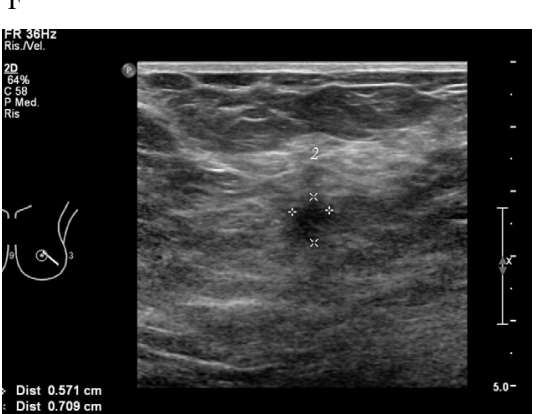

D
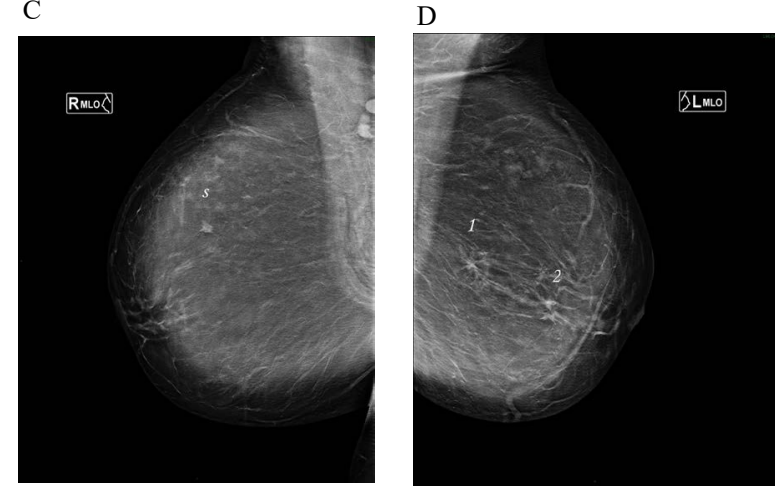

G

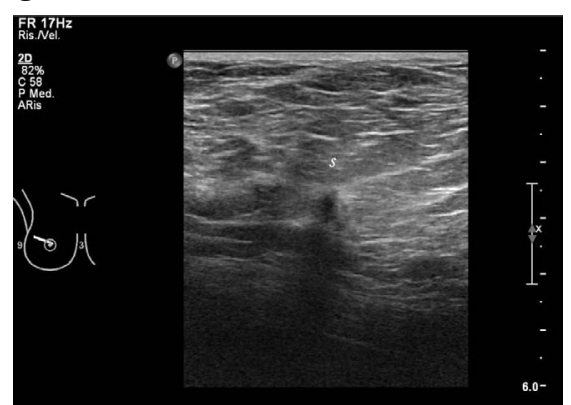

Figure 8. Asymptomatic 55-year-old woman with fatty breast tissue, recalled from FFDM screening for microcalcifications in the left fatty glandular breast. No previous exams available 8A right MLO FFDM, 8B left MLO FFDM, 8C right MLO DBT, 8D left MLO DBT, 8E left US of lesion 1, 8F left US of lesion 2, 8G right US of lesion 3.

MLO FFDM images showed in the left breast (B) two small clusters of microcalcifications associated with architectural distortions.

MLO DBT images (C, D) detected multifocal bilateral disease, with depiction of a small spiculated mass in the contralateral right breast (lesion 3).

US modality $(\mathrm{E}, \mathrm{F}, \mathrm{G})$ confirmed as suspicious all the three lesions.

US-guided core needle biopsy proved multifocal bilateral invasive ductal carcinoma

for evaluation of focal mammographic asymmetry and DBT showed higher sensitivity $(93.8 \%)$ for lesion visualization and differentiation from summation artifacts in comparison to spot compression imaging $(50.2 \%)$

\section{Masses}

Masses are characterized by their shape, margin and density. DBT can depict shape and edges with precise details, underlying a suspicious irregular shape, indistinct or spiculated margins. Zuley et al. [49], found that the use of the word "irregular", as shape adjective, decreased significantly at DBT especially for benign lesions, reclassified at DBT as lobulated or oval.
In some cases, the only clues leading to the detection of a subtle malignant mass are fine spiculations presenting as thin isodense lines secondary to straightening of adjacent fibroglandular tissue and Cooper ligaments: these features are often better depicted at DBT [71], in particular for cancers surrounded by normal fibroglandular tissue, in heterogeneously dense breasts [12] (Figures 4 and 5).

Moreover, DBT can depict iso- or hypodense masses: this is particularly relevant because some cancers are characterized by intratumoral fat, entrapped or absorbed from the surrounding adipose tissue during growth, and appear less radiopaque and badly outlined in FFDM, even in low density breasts [72] (Figure 6). 


\section{Microcalcifications}

Microcalcifications can be the early and unique sign of breast carcinoma. There are many reports regarding the ability of DBT to visualize microcalcifications in comparison with FFDM that is the actual gold standard. Poplack and colleagues [73] compared FFDM with DBT in the evaluation of different lesion subtypes and observed that microcalcification showed better image quality on diagnostic mammography than on tomosynthesis. On the other hand, Kopans and colleagues [74] compared the clarity of microcalcifications on FFDM and DBT, observing that microcalcifications were seen on DBT with better accuracy in $41.6 \%$ of the cases, with equivalent accuracy in $50.4 \%$ and only in $8 \%$ of cases were better seen on FFDM.

Spangler and colleagues [75] demonstrated a statistically significant higher detection rate for calcifications on FFDM than DBT (84\% vs $75 \%)$ and higher specificity in evaluating the calcifications $(71 \%$ vs $64 \%)$. Indeed, if calcifications are small and spread, single reconstructed DBT slices may show only few calcifications of a clinically significant cluster, not allowing a correct spatial evaluation. If calcifications are large, they may produce significant artifacts, appearing on multiple slices as repeating ghost-like, out-of-focus, white objects bordered by dark shadows, marching in the direction of the $\mathrm{x}$-ray tube motion [56].

\section{New perspectives}

Nowadays, studies are focused on evaluating the diagnostic performance of tomosynthesis in screening setting; one example is The Mammographic Imaging Screening Trial (TMIST), which is a randomized breast cancer screening trial that compares FFDM with DBT. The Eastern Cooperative Oncology Group (ECOG) and the American College of Radiology Imaging Network (ACRIN) Cancer Research Group opened the trial on July 6, 2017, and plans to enroll about 165,000 women, aged 45-74 years, by the end of 2020 [76].

One of the major concerns that limit the use of DBT, especially as a screening tool, is the radiant exposure: the introduction of new reconstruction algorithms can potentially solve this issue. There is evidence suggesting that the use of iterative reconstructions allows to obtain better contrast, better image quality, optimal visibility of calcifications and fewer artifacts, compared to traditional filtered back projection reconstructions [77] and to get a better contrast-to-noise ratio with the same radiation dose [78,79].

Further developments are also expected in the area of computeraided detection (CAD): recent studies showed that CAD allows a non-inferiority of reader interpretation performance, with a faster reading time. The introduction of CAD in clinical practice would allow overcoming a limit of tomosynthesis that is the additional reading time $[80,81]$.

Another important innovation is the development of new equipment, like the combination of DBT with automated breast ultrasound: its goal is to implement sensitivity in patients with dense breast; currently, to our knowledge, there are only few data available on small groups of patients in this setting $[82,83]$, but this new technologies would seem to provide promising results.

\section{Conclusions}

Since its recent introduction, DBT is rapidly emerging as a practice changing technique. It improves the accuracy of clinical mammography, by increasing sensitivity and specificity; particularly in screening patients, DBT reduces the recall rates and increases cancer detection. It is a useful tool for further characterization of suspicious findings, improving reading confidence and diagnostic accuracy and for local staging of breast cancer, thus implementing surgical planning.

Although additional data from large multicenter prospective trials and further analysis on cost effectiveness are needed, this technology provides improvement of mammographic imaging and more efficient diagnostic workups.

\section{References}

1. Tabar L, Fagerberg G, Chen HH, Duffy SW, Smart CR, et al. (1995) Efficacy of breast cancer screening by age. New results from the Swedish Two-County Trial. Cancer 75: 2507-2517. [Crossref]

2. Nyström L, Andersson I, Bjurstam N, Frisell J, Nordenskjöld B, et al. (2002) Longterm effects of mammography screening: updated overview of the Swedish randomised trials. Lancet 359:909-919. [Crossref]

3. Berry DA, Cronin KA, Plevritis SK, Fryback DG, Clarke L, et al. (2005) Cancer Intervention and Surveillance Modeling Network (CISNET) Collaborators (2005) Effect of screening and adjuvant therapy on mortality from breast cancer. $N$ Engl $J$ Med 353:1784-1792.

4. Tabár L, Yen AM, Wu WY, Chen SL, Chiu SY, et al. (2015) Insights from the breas cancer screening trials: how screening affects the natural history of breast cancer and implications for evaluating service screening programs. Breast J 21:13-20. [Crossref]

5. Smith RA, Duffy SW, Gabe R, Tabar L, Yen AM, et al. (2004) The randomized trials of breast cancer screening: what have we learned? Radiol Clin North Am 42: 793-806. [Crossref]

6. Webb ML, Cady B, Michaelson JS, Bush DM, Calvillo KZ, et al. (2014) A failure analysis of invasive breast cancer: most deaths from disease occur in women not regularly screened. Cancer 120:2839-2846. [Crossref]

7. Independent UK Panel on Breast Cancer Screening (2012) The benefits and harms of breast cancer screening: an independent review. Lancet 380: 1778-1786. [Crossref]

8. Carney PA, Miglioretti DL, Yankaskas BC, Kerlikowske K, Rosenberg R, et al. (2003) Individual and combined effects of age, breast density, and hormone replacementtherapy use on the accuracy of screening mammography. Ann Intern Med 138:168-175. [Crossref]

9. Day N, Warren R (2000) Mammographic screening and mammographic patterns. Breast Cancer Res 2: 247-251. [Crossref]

10. Kolb TM, Lichy J, Newhouse JH (2002) Comparison of the performance of screening mammography, physical examination, and breast US and evaluation of factors that influence them: an analysis of 27,825 patient evaluations. Radiology 225: 165-175. [Crossref]

11. Rosenberg RD, Hunt WC, Williamson MR, Gilliland FD, Wiest PW, et al. (1998) Effects of age, breast density, ethnicity, and estrogen replacement therapy on screening mammographic sensitivity and cancer stage at diagnosis: review of 183,134 screening mammograms in Albuquerque, New Mexico. Radiology 209:511-518.

12. AL Mousa DS, Ryan EA, Mello-Thoms C, Brennan PC (2014) What effect does mammographic breast density have on lesion detection in digital mammography? Clin Radiol 69:333-341.

13. Kolb TM, Lichy J, Newhouse JH (2002) Comparison of the performance of screening mammography, physical examination, and breast US and evaluation of factors that influence them: an analysis of 27,825 patient evaluations. Radiology 225:165-175.

14. Mandelson MT, Oestreicher N, Porter PL, White D, Finder CA, et al. (2000) Breas density as a predictor of mammographic detection: comparison of interval- and screendetected cancers. J Natl Cancer Inst 92:1081-1087

15. Duncan KA, Needham G, Gilbert FJ, Deans HE (1998) Incident round cancers: what lessons can we learn? Clin Radiol 53: 29-32. [Crossref]

16. Michell MJ1 (2012) Breast screening review--a radiologist's perspective. $B r J$ Radiol 85: 845-847. [Crossref]

17. Rowlands JA, Hunter DM, Araj N (1991) X-ray imaging using amorphous selenium: a photoinduced discharge readout method for digital mammography. Med Phys 18: 421431. [Crossref]

18. Vedantham S, Karellas A, Suryanarayanan S, Albagli D, Han S, et al. (2000) Full breas digital mammography with an amorphous silicon-based flat panel detector: physical characteristics of a clinical prototype. Med Phys 27:558-567 
19. Pisano ED, Gatsonis C, Hendrick E, Yaffe M, Baum JK, et al. Digital Mammographic ImagingScreening Trial (DMIST) Investigators Group (2005) Diagnostic performance of digital versus film mammography for breast-cancer screening. $N$ Engl J Med 353:1773-1783

20. Gilbert FJ, Tucker L, Young KC (2016) Digital breast tomosynthesis (DBT): a review of the evidence for use as a screening tool. Clin Radiol 71: 141-150. [Crossref]

21. Hooley RJ, Durand MA, Philpotts LE (2017) Advances in Digital Breast Tomosynthesis. AJR Am J Roentgenol 208: 256-266. [Crossref]

22. Lei J, Yang P, Zhang L, Wang Y, Yang K (2014) Diagnostic accuracy of digital breast tomosynthesis versus digital mammography for benign and malignant lesions in breasts: a meta-analysis. Eur Radiol 24: 595-602. [Crossref]

23. Andersson I, Ikeda DM, Zackrisson S, Ruschin M, Svahn T, Timberg P (2008) Breas tomosynthesis and digital mammography: a comparison of breast cancer visibility and BIRADS classification in a population of cancers with subtle mammographic findings. Eur Radiol 18:2817-2825.

24. Dobbins JT 3rd1, Godfrey DJ (2003) Digital x-ray tomosynthesis: current state of the art and clinical potential. Phys Med Biol 48: R65-106. [Crossref]

25. Dobbins JT 3rd1 (2009) Tomosynthesis imaging: at a translational crossroads. Med Phys 36: 1956-1967. [Crossref]

26. Sechopoulos I (2013) A review of breast tomosynthesis. Part I. The image acquisition process. Med Phys 40: 014301. [Crossref]

27. Roth RG, Maidment ADA, Weinstein SP, Roth SO, Conant EF (2014) Digital breast tomosynthesis: lessons learned from early clinical implementation. RadioGraphics 34 : E89-102.

28. Peppard HR, Nicholson BE, Rochman CM, Merchant JK, Mayo RC 3rd, et al. (2015) Digital Breast Tomosynthesis in the Diagnostic Setting: Indications and Clinical Applications. Radiographics 35:975-990.

29. Hardesty LA (2015) Issues to consider before implementing digital breast tomosynthesis into a breast imaging practice. AJR Am J Roentgenol 204:681-684.

30. Acciavatti RJ, Maidment AD (2012) Observation of super-resolution in digital breast tomosynthesis. Med Phys 39: 7518-7539. [Crossref]

31. United States Food and Drug Administration. MQSA facility certification extension requirements for digital breast tomosynthesis (DBT) system. https://www.fda.gov/ Radiation-EmittingProducts/MammographyQualityStandardsActandProgram/Facility CertificationandInspection/ucm413117.htm [Accessed 14 June 2018]

32. Bouwman RW, van Engen RE, Young KC, den Heeten GJ, Broeders MJ, et al. (2015) Average glandular dose in digital mammography and digital breast tomosynthesis: comparison of phantom and patient data. Phys Med Biol 60:7893-7907.

33. Svahn TM, Houssami N, Sechopoulos I, Mattsson S (2015) Review of radiation dose estimates in digital breast tomosynthesis relative to those in two-view full-field digital mammography. Breast 24:93-99.

34. Feng SS, Sechopoulos I (2012) Clinical digital breast tomosynthesis system: dosimetric characterization. Radiology 263:35-42.

35. Maldera A, De Marco P, Colombo PE, Origgi D, Torresin A (2017) Digital breast tomosynthesis: Dose and image quality assessment. Phys Med 33: 56-67. [Crossref]

36. European Reference Organization for Quality Assured Breast Screening and Diagnostic Service (2006) European guidelines for quality assurance in breast cancer screening and diagnosis. 4 th ed

37. Durand MA, Wang S, Hooley RJ, Raghu M, Philpotts LE (2016) Tomosynthesisdetected Architectural Distortion: Management Algorithm with Radiologic-Pathologic Correlation. Radiographics 36:311-321.

38. Shin SU, Chang JM, Bae MS, Lee SH, Cho N, et al. (2015) Comparative evaluation of average glandular dose and breast cancer detection between single-view digital breast tomosynthesis (DBT) plus single-view digital mammography (DM) and two-view DM: correlation with breast thickness and density. Eur Radiol 25:1-8.

39. Vedantham S, Karellas A, Vijayaraghavan GR, Kopans DB (2015) Digital Breast Tomosynthesis: State of the Art. Radiology 277: 663-684. [Crossref]

40. Baker JA, Lo JY (2011) Breast tomosynthesis: state-of-the-art and review of the literature. Acad Radiol 18: 1298-1310. [Crossref]

41. Helvie MA (2010) Digital mammography imaging: breast tomosynthesis and advanced applications. Radiol Clin North Am 48: 917-929.

42. Wallis MG, Moa E, Zanca F, Leifland K, Danielsson M (2012) Two-view and singleview tomosynthesis versus full-field digital mammography: high-resolution X-ray imaging observer study. Radiology 262:788-796.
43. U.S. Food Drug Administration, P080003/S001 Hologic Selenia Dimensions 3D System, FDA Executive Summary, FDA, 2012.

44. Zuley ML, Guo B, Catullo VJ, Chough DM, Kelly AE, et al. (2014) Comparison of two-dimensional synthesized mammograms versus original digital mammograms alone and in combination with tomosynthesis images. Radiology 271:664-671.

45. Nelson JS, Wells JR, Baker JA, Samei E (2016) How does c-view image quality compare with conventional 2D FFDM? Med Phys 43: 2538. [Crossref]

46. Zuckerman SP, Maidment ADA, Weinstein SP, McDonald ES, Conant EF (2017) Imaging with Synthesized 2D Mammography: Differences, Advantages, and Pitfalls Compared with Digital Mammography. AJR Am J Roentgenol 209:222-229.

47. Dang PA, Freer PE, Humphrey KL, Halpern EF, Rafferty EA (2014) Addition of tomosynthesis to conventional digital mammography: effect on image interpretation time of screening examinations. Radiology 270: 49-56.

48. Skaane P, Bandos AI, Gullien R, Eben EB, Ekseth U, et al. (2013) Comparison of digital mammography alone and digital mammography plus tomosynthesis in a populationbased screening program. Radiology 267:47-56.

49. Zuley ML, Bandos AI, Ganott MA, Sumkin JH, Kelly AE, (2013) Digital breas tomosynthesis versus supplemental diagnostic mammographic views for evaluation of noncalcified breast lesions. Radiology 266:89-95.

50. Lourenco AP, Barry-Brooks M, Baird GL, Tuttle A, Mainiero MB (2015) Changes in recall type and patient treatment following implementation of screening digital breast tomosynthesis. Radiology 274:337-342.

51. Rose SL, Tidwell AL, Bujnoch LJ, Kushwaha AC, Nordmann AS, et al. (2013) Implementation of breast tomosynthesis in a routine screening practice: an observational study. AJR Am J Roentgenol 200:1401-1408.

52. McCarthy AM, Kontos D, Synnestvedt M, Tan KS, Heitjan DF, et al. (2014) Screening outcomes following implementation of digital breast tomosynthesis in a generalpopulation screening program. J Natl Cancer Inst. 106(11).

53. Greenberg JS, Javitt MC, Katzen J, Michael S, Holland AE (2014) Clinical performance metrics of 3D digital breast tomosynthesis compared with 2D digital mammography for breast cancer screening in community practice. AJR Am J Roentgenol 203:687-93.

54. Sharpe RE Jr, Venkataraman S, Phillips J, Dialani V, Fein-Zachary VJ, et al. (2016) Increased Cancer Detection Rate and Variations in the Recall Rate Resulting from Implementation of 3D Digital Breast Tomosynthesis into a Population-based Screening Program. Radiology 278: 698-706.

55. Friedewald SM, Rafferty EA, Rose SL, Durand MA, Plecha DM, et al. (2014) Breast cancer screening using tomosynthesis in combination with digital mammography. JAMA 311:2499-2507.

56. Conant EF (2014) Clinical implementation of digital breast tomosynthesis. Radiol Clin North Am 52:499-518.

57. Spangler ML, Zuley ML, Sumkin JH, Abrams G, Ganott MA, et al. (2011) Detection and classification of calcifications on digital breast tomosynthesis and 2D digital mammography: a comparison. AJR Am J Roentgenol 196: 320-324. [Crossref]

58. Brandt KR, Craig DA, Hoskins TL, Henrichsen TL, Bendel EC, et al. (2013) Can digital breast tomosynthesis replace conventional diagnostic mammography views for screening recalls without calcifications? A comparison study in a simulated clinical setting. AJR Am J Roentgenol 200:291-298.

59. Tagliafico A, Astengo D, Cavagnetto F, Rosasco R, Rescinito G, et al. (2012) One-to-one comparison between digital spot compression view and digital breast tomosynthesis. Eur Radiol 22:539-544.

60. Houssami N, Skaane P (2013) Overview of the evidence on digital breast tomosynthesis in breast cancer detection. Breast 22:101-8.

61. Noroozian M, Hadjiiski L, Rahnama-Moghadam S, Klein KA, Jeffries DO, et al. (2012) Digital breast tomosynthesis is comparable to mammographic spot views for mas characterization. Radiology 262:61-68.

62. Garayoa J, Chevalier M, Castillo M, Mahillo-Fernández I, Amallal E, et al. (2018) Diagnostic value of the stand-alonesynthetic image in digital breast tomosynthesis examinations. Eur Radiol 28:565-572.

63. Bernardi D, Belli P, Benelli E, Brancato B, Bucchi L, et al. (2017) Digital breast tomosynthesis (DBT): recommendations from the Italian College of Breast Radiologists (ICBR) by the Italian Society of Medical Radiology (SIRM) and theItalian Group for Mammography Screening (GISMa). Radiol Med 122:723-730.

64. Leung JW1, Sickles EA (2000) Multiple bilateral masses detected on screening mammography: assessment of need for recall imaging. AJR Am J Roentgenol 175: 2329. [Crossref] 
65. Menezes GL, Knuttel FM, Stehouwer BL, Pijnappel RM, van den Bosch MA (2014) Magnetic resonance imaging in breast cancer: A literature review and future perspectives. World J Clin Oncol 5:61-70.

66. Luparia A, Mariscotti G, Durando M, Ciatto S, Bosco D, et al. (2013) Accuracy of tumour size assessment in the preoperative staging of breast cancer: comparison of digital mammography, tomosynthesis, ultrasound and MRI. Radiol Med 118:11191136.

67. Harvey JA, March DE (2013) Making the diagnosis: a practical guide to breast imaging. Philadelphia, Pa: Elsevier Saunders.

68. Dominguez A, Durando M, Mariscotti G, Angelino F, Castellano I, et al. (2015) Breast cancer risk associated with the diagnosis of a microhistological radial scar (RS) retrospective analysis in 10 years of experience. Radiol Med 120:377-385.

69. D’Orsi CJ, Sickles EA, Mendelson EB, Morris EA (2013) Breast Imaging and Reporting Data System: ACR BI-RADS breast imaging atlas. Reston, Va: American College of Radiology

70. Bahrs SD, Otto V, Hattermann V, Klumpp B, Hahn M, et al. (2018) Breast tomosynthesis for the clarification of mammographic BI-RADS 3 lesions can decrease follow-up examinations and enables immediate cancer diagnosis. Acta Radiol 59:1176-1183.

71. Luparia A, Mariscotti G, Durando M, Ciatto S, Bosco D, et al. (2013) Accuracy of tumour size assessment in the preoperative staging of breast cancer: comparison of digital mammography, tomosynthesis, ultrasound and MRI. Radiol Med 118:11191136.

72. Freer PE, Wang JL, Rafferty EA (2014) Digital breast tomosynthesis in the analysis of fat-containing lesions. Radiographics 34: 343-358. [Crossref]

73. Poplack SP, Tosteson TD, Kogel CA, Nagy HM (2007) Digital breast tomosynthesis: Initial experience in 98 women with abnormal digital screening mammography. AJR Am J Roentgenol 189:616-623

74. Kopans D, Gavenonis S, Halpern E, Moore R (2011) Calcifications in the breast and digital breast tomosynthesis. Breast $J$ 17:638-644.
75. Spangler ML, Zuley M, Sumkin J (2011) Detection and classification of calcifications on digital breast tomosynthesis and 2D digital mammography: a comparison. AJR Am J Roentgenol 196:320-324.

76. ECOG-ACRIN cancer research group. TMIST Breast Cancer Screening Trial, http:// ecog-acrin.org/tmist/; 2018 [accessed 14 June 2018]

77. Rodriguez-Ruiz A, Teuwen J, Vreemann S, Bouwman RW, van Engen RE, et al. (2017) New reconstruction algorithm for digital breast tomosynthesis: better image quality for humans and computers. Acta Radiol 1:284185117748487.

78. Polat A, Yildirim I (2018) An iterative reconstruction algorithm for digital breast tomosynthesis imaging using real data at three radiation doses. J Xray Sci Technol 26:347-360.

79. Kim YS, Park HS, Lee HH, Choi YW, Choi JG, et al. (2016) Comparison study ofreconstruction algorithms for prototype digital breast tomosynthesis usingvarious breast phantoms. Radiol Med 121:81-92.

80. Balleyguier C, Arfi-Rouche J, Levy L, Toubiana PR, Cohen-Scali F, et al. (2017) Improving digital breast tomosynthesis reading time: A pilot multi-reader, multi-case study using concurrent Computer-Aided Detection (CAD). Eur J Radiol 97:83-89.

81. Benedikt RA, Boatsman JE, Swann CA, Kirkpatrick AD, Toledano AY (2018) Concurrent Computer-Aided Detection Improves Reading Time of Digital Breast Tomosynthesis and Maintains Interpretation Performance in a Multireader Multicase Study. AJR Am J Roentgenol 210:685-694.

82. Schaefgen B, Heil J, Barr RG, Radicke M, Harcos A, et al. (2018) Initial results of the FUSION-X-US prototype combining $3 \mathrm{D}$ automated breast ultrasound and digital breast tomosynthesis. Eur Radiol 28:2499-2506.

83. Larson ED, Lee WM, Roubidoux MA, Goodsitt MM, Lashbrook C, et al. (2018) Preliminary Clinical Experience with a Combined Automated Breast Ultrasound and Digital Breast Tomosynthesis System. Ultrasound Med Biol 44:734-742.

Copyright: $\odot 2018$ Marcello Alessandro ORSI. This is an open-access article distributed under the terms of the Creative Commons Attribution License, which permits unrestricted use, distribution, and reproduction in any medium, provided the original author and source are credited. 